kleare Wechselwirkungen maßgebend sind $^{1}$. Nimmt man z. B. beim ${ }_{80}^{200} \mathrm{Hg}$-Kern die in Abb. 3 angedeutete Ladungsverteilung an, dann zeigt die einfache Durchrechnung mittels der Bornschen Näherung, daß diese Ladungsverteilung für die Streuung von Elektronen äquivalent ist einer homogenen Ladungsverteilung (bei derselben Gesamtladung) mit dem Radius $R_{\mathrm{e}}=R+$ $1 / 2 \Delta R \approx 8 \cdot 10^{-13} \mathrm{~cm}$. Dasselbe gilt für die Radiusausmessung mittels des Spektrums der $\mu$-Mesonatome, da hierbei dasselbe maßgebende Wechselwirkungsintegral auftritt. Bei der Radiusausmessung mittels Streuung schneller Nukleonen ist dagegen zu erwarten, daß wegen der starken nuklearen Wechselwirkung (Bohr- sches Sandsackmodell) auch noch eine ziemlich weit außen liegende Randzone bzgl. Nukleoneneinfang und unelastischer Streuung als schwarz erscheint. Nehmen wir z. B. an, daß der Kernradius, der durch die Nukleonenstreuung bestimmt wird, $R_{\text {Nukl }}=R+4 / 5 \Delta R$ sei, so ergibt sich ein

$$
\Delta R_{0} \mathrm{III} \approx 0,1 \cdot 10^{-13} \mathrm{~cm} .
$$

D. h., das gesamte resultierende $\Delta R_{0}$ res. kommt in die richtige Größenordnung von $2 \cdot 10^{-13} \mathrm{~cm}$. Genauere Untersuchungen $\mathrm{zu}$ den eben gebrachten groben $\mathbf{A b}$ schätzungen werden vorbereitet.

\title{
NOTIZEN
}

\section{Über die Halbleitereigenschaften von Galliumphosphid}

Von O. G. Folberth und F. Oswald

Aus dem Forschungslaboratorium der SiemensSchuckertwerke AG. Erlangen

(Z. Naturforschg. 9a, 1050-1051 [1954]; eingeg. am 1. November 1954)

Die Breite der verbotenen Zone der im Zinkblendegitter kristallisierenden Halbleiter vom Typus $\mathrm{A}^{\mathrm{III}} \mathrm{B}^{\mathrm{V}}$ nimmt mit abnehmender Ordnungszahl der Komponenten $\mathrm{zu}^{1}$. Bei Galliumphosphid (GaP) ist deshalb ein größerer Bandabstand zu erwarten als bei Galliumarsenid $(1,35 \mathrm{eV})$ bzw. Indiumphosphid $(1,25 \mathrm{eV})^{2}$.

Die Verbindung wurde erstmals von V. M. Goldschmidt ${ }^{3}$ durch Erhitzen von Galliumhydroxyd in einem mit Phosphor-Dampf gesättigten Wasserstoffstrom bei etwa $500^{\circ} \mathrm{C}$ hergestellt. Die Strukturbestimmung der entstandenen feinkristallin gesinterten Masse ergab ein Gitter des Zinkblendetyps mit einer Kantenlänge der Elementarzelle von $a=5,436 \AA$.

Zur Bestimmung der optischen und elektrischen Halbleitereigenschaften sind jedoch größere, homogene, gutkristallisierte Bereiche der Substanz notwendig. Durch Zusammenschmelzen der Elemente im abgeschmolzenen Quarzrohr und anschließendes langsames, gerichtetes Erstarren der Schmelze ist es gelungen, Galliumphosphid der gewünschten Güte zu erhalten. Die Verbindung ist orange gefärbt, durchsichtig und zeigt bei streifender Betrachtung metallischen Glanz.

Zur Bestimmung der Lage der Absorptionskante, welche in enger Beziehung zur Breite der verbotenen Zone steht, wurde die Lichtdurchlässigkeit von planparallelen, polierten Proben von 0,1 bis $1,0 \mathrm{~mm}$ Dicke mit einem registrierenden Spektralphotometer im Wellenlängenbereich von 0,5 bis $15 \mu$ aufgenommen. Die Absorptionskante, bei allen bisher hergestellten AIII$\mathrm{BV}^{\mathrm{V}}$-Verbindungen im infraroten Spektralbereich liegend $^{2}$, ist hier in das sichtbare Gebiet gerückt. Durch

${ }^{1}$ H. Welker, Z. Naturforschg. 7a, 744 [1952]; 8a, 248 [1953].

2 F. Oswald u. R. Schade, Z. Naturforschg. 9a, 611 [1954].
Messungen bei verschiedenen Temperaturen im Bereich von etwa $100^{\circ} \mathrm{K}$ bis $500^{\circ} \mathrm{K}$ konnte die Verschiebung der Kante mit der Temperatur bestimmt werden. Dabei ergab sich für die Lage der Kante (ausgedrückt in Elektronenvolt) eine lineare Abhängigkeit von der Temperatur $T$, die sich in dem gemessenen Bereich darstellen läßt durch

$$
\Delta E=2,4-5,5 \cdot 10^{-4} \cdot T[\mathrm{eV}] .
$$

Die Lage der Absorptionskante am absoluten Nullpunkt $(2,4 \mathrm{eV})$ wurde durch Extrapolation ermittelt.

Die Durchlässigkeitsmessungen wurden noch durch Aufnahme des Reflexionsvermögens der Substanz in dem genannten Spektralbereich ergänzt. Aus beiden Größen ließen sich dann nach dem in einer früheren Arbeit ${ }^{2}$ angegebenen Verfahren die optischen Konstanten (Absorptionskonstante $K$ und Brechungsindex $n$ ) von Galliumphosphid berechnen (Abb. 1). Im Bereich

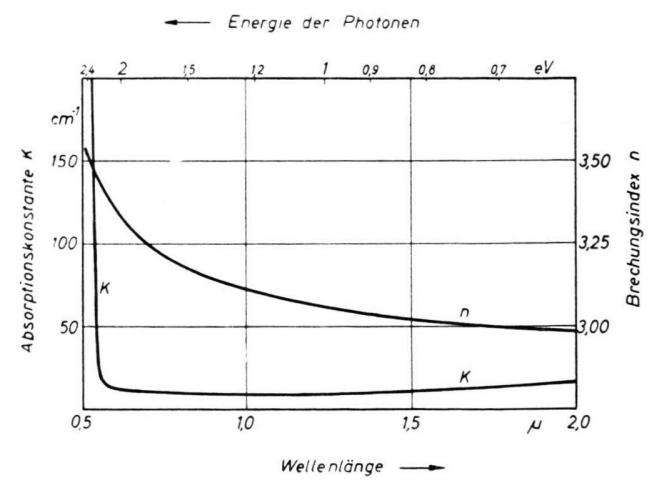

Abb. 1.

von 0,51 bis $0,77 \mu$ wurde der Brechungsindex außerdem durch Messungen der Lichtbrechung an einem Galliumphosphid-Prisma von etwa $15^{\circ}$ brechendem Winkel und $1 \mathrm{~mm}$ Basisbreite bestimmt. In diesem

3 Gmelins Handbuch der anorg. Chemie, System Nr. 36, 95 [1936]. 
Bereich zeigt die untersuchte Substanz einen für durchsichtige Medien ungewöhnlich hohen Brechungsindex $\left(n_{\mathrm{D}}=3,37\right)$ und sehr hohe Dispersion $(\mathrm{d} n / \mathrm{d} \lambda)_{\mathrm{D}} \approx$ $-1,6 \cdot 10^{4} \mathrm{~cm}^{-1}$, so daß die Na-D-Linien von dem benützten Prisma noch getrennt werden. Im infraroten Spektralgebiet nimmt der Brechungsindex auf etwa 2,9 und die Dispersion auf weniger als $-1 \cdot 10^{2} \mathrm{~cm}^{-1}$ ab. Die Absorptionskonstante durchläuft ein Minimum bei etwa $1 \mu$ und steigt nach längeren Wellen wieder an.
Die erschmolzenen Proben waren im allgemeinen p-leitend, durch Dotieren der Schmelze mit Schwefel konnten jedoch auch n-leitende Kristalle hergestellt werden.

Nach freundlicher Mitteilung von Herrn Dr. R. Gremmelmaier zeigt Galliumphosphid Spitzengleichrichterwirkung; außerdem wurde ein lichtelektrischer Effekt festgestellt.

Über die elektrischen Eigenschaften von Galliumphosphid wird noch ausführlich berichtet werden.

\section{Über die Erhaltung der Fermionenladung}

Von G. Marx

Institut für Physik der Roland Eötvös Universität, Budapest

(Z. Naturforschg. 9a, 1051-1052 [1954]; eingeg. am 26. Oktober 1954)

Im letzten Jahrzehnt trat an die Stelle der Annahme von der Unveränderlichkeit der Elementarteilchen die Erkenntnis, daß die Neigung, sich umzuwandeln — die Instabilität - eher eine Regel als eine Ausnahme bei den Elementarteilchen darstellt. Man kann sagen, daß sie jede Umwandlung erleiden, die nicht durch irgendein physikalisches Gesetz (Erhaltungssatz) untersagt wird. Hier sind die dynamischen Sätze (Erhaltungssätze von Energie, Impuls und Drehimpuls) sowie der Erhaltungssatz der elektrischen Ladung zu erwähnen. Bei der Untersuchung der zwischen den Elementarteilchen vorkommenden Umwandlungen folgerte Wig ner in den letzten Jahren das Bestehen eines weiteren Erhaltungssatzes, der in der klassischen Physik unbekannt war, nämlich die Erhaltung der Nukleonenladung 1, 2. Dieses Gesetz erklärt die Beobachtung, daß die Zahl der Nukleonen bei den Umwandlungen unverändert bleibt, sowie die Gleichheit der zwischen den verschiedenen Nukleonen wirkenden Kernkräfte (vgl. l. c. ${ }^{3,4}$ ).

Mehrere Forscher wiesen darauf hin, daß die oben erwähnten Erhaltungssätze keine befriedigende Erklärung für sämtliche Gesetzmäßigkeiten geben, die sich bei den Wechselwirkungen der Elementarteilchen beobachten lassen ${ }^{5}$. Es ist bekannt, daß die Kopplungskonstante der zwischen den vier Fermionen auftretenden Wechselwirkungen ( $\beta$-Zerfall und K-Einfang, Zerfall bzw. Einfang des $\mu$-Mesons) innerhalb der empirischen und theoretischen Genauigkeit von gleicher Größe ist ${ }^{6}$. Klein zog auf ähnlicher Grundlage den Schluß, daß zwischen vier beliebigen Fermionen stets eine universale Fermionenwechselwirkung besteht ${ }^{7}$. Aus dieser Annahme würden sich allerdings auch Zerfallsprozesse ergeben, die man in der Natur nicht beobachten kann ${ }^{8,9}$.

1 E. Wigner, Proc. Amer. Phil. Soc. 93, 521 [1949].

2 E. Wigner, Proc. Nat. Akad. Sci. 38, 449 [1952].

${ }^{3}$ L. I. Schiff, Phys. Rev. 85, 374 [1952].

4 A. B. Seldowitsch, Dokl. Akad. Nauk SSSR 86, 505 [1952].

5 E. Fermi, Elementary Particles, 1951.

6 Tiomno-Wheeler, Rev. Mod. Phys. 21, 153 [1949].
Zur Erklärung der erwähnten Erscheinungen folgerten der Verfasser ${ }^{10}$ und, unabhängig von ihm und in einer etwas anderen Form, Seld owits $\mathrm{ch}^{8}$ einen neuen Erhaltungssatz. Nach dieser Annahme besitzt jedes Fermion (außer der eventuellen elektrischen und $\mathrm{Nu}$ kleonenladung) eine sog. Fermionenladung, und auch diese Ladung bleibt bei allen Umwandlungen erhalten. (In der Fassung von Seldowitsch heiß es Neutrinoladung, und nach ihm besitzen nur die leichten Fermionen - Neutrino, Elektron-Positron, $\mu$-Meson - eine Neutrinoladung.) Berücksichtigt man den eingeführten Erhaltungssatz und die Fähigkeit der Fermionen, sich ineinander umzuwandeln, so gelangt man zur Folgerung, daß der Zahlenwert der Ladung sämtlicher Fermionen gleich ist, und zwar beträgt die Ladung der ,wirklichen" Teilchen $+f$ und die der im Diracschen Sinn genommenen Antiteilchen - f. Seldowitsch betrachtet den Zahlenwert von $f$ als durch Messung unbestimmbar. Nach Ansicht des Verfassers wird die Stärke der Fermionenwechselwirkung durch die $f$-Ladung bestimmt. Die Fermische Wechselwirkungskonstante $F$ des $\beta$-Zerfalls und anderer Prozesse ergibt sich aus dem Produkt der $f$-Ladungen der an der Wechselwirkung teilnehmenden vier Fermionen. Die aus dem Erhaltungssatz folgende Gleichheit der $f$-Ladungen gibt die Erklärung für die beobachtete Übereinstimmung der Wechselwirkungskonstante $F$ bei den verschiedenen Prozessen. Zieht man die aus Messungen ermittelte Größe der Kopplungskonstante $F$ in Betracht, so erhält man einen Wert von ungefähr

$$
\begin{aligned}
f & =F^{1 / 4}=\left(5 \cdot 10^{49} \mathrm{erg} \mathrm{cm}^{3}\right)^{1 / 4} \\
& =2,5 \cdot 10^{-12} \mathrm{~cm}^{3 / 4} \mathrm{~g}^{1 / 4} \mathrm{sec}^{1 / 2} .
\end{aligned}
$$

Mit dem Erhaltungssatz der Fermionenladung läßt sich auch jene von Konopinski und Mahmoud empirisch aufgestellte Forderung ${ }^{9}$ erklären, daß der Hamilton-Operator der universalen Fermionenwechselwirkung die Form

$$
H=F \int\left(\psi_{1}^{*} \alpha \psi_{\mathrm{z}}\right)\left(\psi_{3} * \alpha \psi_{4}\right) \mathrm{d} x
$$

haben müsse. In dieser Gleichung bedeutet $\psi_{i}$ einen die Absorption eines Fermions (die Emission eines Antifermions) und $\psi_{j}{ }^{*}$ einen die Emission eines Fer-

7 O. Klein, Nature, Lond. 161, 897 [1948].

8 A.B. Seldowitsch, Dokl. Akad. Nauk SSSR 91, 1317 [1953].

${ }^{9}$ E. J. Konopinski u. H. M. Mahmoud, Phys. Rev. 92, 1045 [1953].

10 G. Marx, Acta Phys. Hung. 3, 55 [1953]. 